\title{
The PIDDosome, DNA-damage-induced apoptosis and beyond
}

\author{
S Janssens ${ }^{\star, 1}$ and A Tinel ${ }^{\star, 2}$
}

P53-induced protein with a death domain (PIDD) was cloned as a death domain (DD)-containing protein whose expression is induced by $\mathrm{p} 53$. It was later described as the core of a molecular platform-activating caspase-2, named the PIDDosome. These first results pointed towards a role for PIDD in apoptosis, in response to DNA damage. Identification of new PIDDosome complexes involved in DNA repair and nuclear factor- $\kappa \mathrm{B}$ signaling challenged this early concept. PIDD functions are growing as new complexes and new interaction partners are being discovered, and as additional functions are being revealed. A fascinating feature of PIDD lies within its complex and tight regulation mechanisms, which allow the molecule to fine-tune its different functions: from transcriptional regulation to the expression of different isoforms, and from the interaction with regulatory proteins to an ingenious post-translational cleavage mechanism generating various active fragments with specific functions. Further studies still need to be carried out to provide answers to many unresolved issues and to reconcile conflicting results. This review aims at providing an overview of the current PIDD knowledge status.

Cell Death and Differentiation (2012) 19, 13-20; doi:10.1038/cdd.2011.162; published online 18 November 2011

\section{Facts}

- P53-induced protein with a death domain (PIDD) has been identified as a p53-inducible molecule implicated in p53-dependent apoptosis.

- PIDD has a similar architectural structure as other caspaseactivating molecules such as ASC or apoptotic proteaseactivating factor 1 (Apaf-1) and forms the core of a large PIDDosome complex.

- PIDDosome complexes exist within the cell with different molecular compositions and different biological function.

- PIDD function is tightly regulated, its most prominent feature being the sequential generation of a prosurvival and a proapoptotic fragment through an intein-like autoprocessing mechanism.

\section{Open Questions}

- Is PIDD playing a prominent role in cell cycle arrest and/or DNA repair rather than apoptosis? Does PIDD main function remain to be identified?
- Is PIDD involved at all in caspase-2 activation (which is distinct from processing) in vivo? Are both proteins operating in the same pathway or acting independently of each other?

- Identification of molecular activators of PIDD.

PIDD, originally known as LRDD (leucine-rich repeat and death domain-containing protein), was both picked out of a bioinformatics screen searching for novel death domain (DD)containing proteins, ${ }^{1}$ as well as independently identified as a p53-inducible gene (hence its name) in an erythroleukemia cell line expressing a temperature-sensitive p53 mutant allele. $^{2}$ PIDD is located at chromosome 11 p15.5 in humans and is conserved in Vertebrata. It is widely expressed with the highest expression levels found in the spleen and kidney. The protein consists of an $\mathrm{N}$-terminal region with seven leucinerich repeats (LRR), followed by two ZU-5 domains and a C-terminal death domain (DD) (see Figure 1). It shares this architecture with members of the ankyrin, UNC5 and ZUD (Zu5 and DD-containing inhibitor of NF- $\kappa$ B) family that also have a central ZU-5 domain and a C-terminal DD. Recently, another domain called uncharacterized protein domain in

\footnotetext{
${ }^{1}$ GROUP-ID Consortium, Laboratory for Immunoregulation and Mucosal Immunology, Department of Pulmonary Medicine, University Hospital of Ghent, De Pintelaan 185-B, Ghent 9000, Belgium and ${ }^{2}$ Debiopharm SA, Forum Après-Demain, Chemin de Messidor 5-7, Lausanne 1002, Switzerland

*Corresponding author: S Janssens, GROUP-ID Consortium, Laboratory for Immunoregulation and Mucosal Immunology, Department of Pulmonary Medicine, University Hospital of Ghent, De Pintelaan 185-B, Ghent 9000, Belgium. Tel: + 329332 9808; Fax: + 329332 9476; E-mail: Sophie.Janssens@UGent.be or A Tinel, Debiopharm SA, Forum Après-Demain, Chemin de Messidor 5-7, Lausanne 1002, Switzerland. Tel: + 41 (0)21 3210111 ; Fax: + 41 (0)21 321 0169; E-mail: atinel@ debiopharm.com

Keywords: PIDD; caspase-2; PIDDosome; DNA damage; apoptosis

Abbreviations: Apaf-1, apoptotic protease-activating factor 1; CARD, caspase activation and recruitment domain; Chk1, checkpoint kinase 1; DD, death domain; $\mathrm{ER}$, endoplasmic reticulum; IAP, inhibitor of apoptosis protein; $\mathrm{kDa}$, kiloDalton; LRDD, leucine-rich repeats containing protein with a death domain; LRR, leucine-rich repeats; INF- $\kappa$ B, nuclear factor- $\kappa B$; NLR, Nod-like receptors; NLRP3, Nod-like receptors protein 3; PCNA, proliferating cell nuclear antigen; PIDD, p53-induced protein with a death domain; RAIDD, RIP-associated ICH-1/CED-3 homologous protein with a death domain; RIP, receptor-interacting protein; siRNA, small interfering RNA; UPA, uncharacterized protein domain in UNC5, PIDD and Ankyrin family of proteins; VAD-fmk, N-benzyloxycarbonyl-Val-Ala-Asp-fluoromethylketone; ZUD, Zu5 and DD-containing inhibitor of NF- $\kappa \mathrm{B}$

Received 10.8.11; revised 14.10.11; accepted 17.10.11; Edited by G Melino; published online 18.11.11
} 


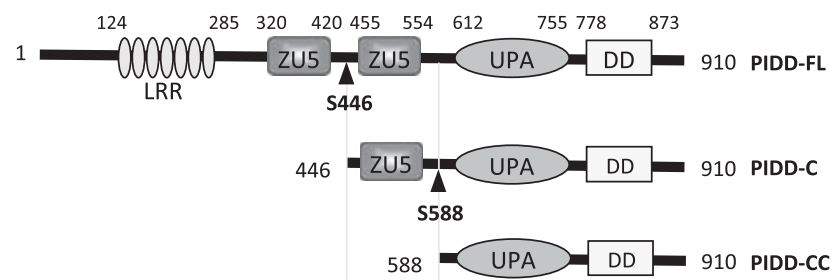

$1-\bigcup_{\text {LRR }}$ OUOD $=445$

PIDD-N

Figure 1 Schematic representation of PIDD and of the fragments resulting from the autoprocessing. Amino-acid positions of each domain are indicated

UNC5, PIDD and Ankyrin family of proteins (UPA) (for uncharacterized protein domain in UNC5, PIDD and Ankyrin family of proteins) was identified between the ZU-5 and the DD. ${ }^{3}$ The 910 amino acid containing full-length PIDD, running at $101 \mathrm{kDa}$, is constitutively cleaved, giving rise to an N-terminal fragment of approximately $48 \mathrm{kDa}$ (called PIDD-N), and two C-terminal fragments running at $55 \mathrm{kDa}$ (called PIDD-C) and at $37 \mathrm{kDa}$ (called PIDD-CC). ${ }^{1,4,5}$ Cleavage sites were defined at $\mathrm{S} 446$ and $5588 .{ }^{6}$ In resting cells, PIDD elutes in the $\sim 500 \mathrm{kDa}$ fraction of a gel filtration experiment, suggesting that it is either in a complex with other proteins or constitutively oligomerized. ${ }^{4}$ The recently identified UPA domain residing between residues 612 and 755 may be responsible for PIDD oligomerization. ${ }^{3}$ PIDD characterization really took off when it was described as the core of a molecular platform-activating caspase-2, the PIDDosome. In agreement with an early report, ${ }^{2}$ these results suggested a role for PIDD in DNA damageinduced apoptosis. Further studies led to the identification of additional PIDDosome complexes with a different composition. In this review, we will describe the different PIDDosome complexes identified so far and summarize their known biological functions.

\section{PIDD and Apoptosis: the Caspase-2 PIDDosome}

Initiator caspases are activated in multicomponent protein complexes such as the Apaf-1-containing apoptosome for caspase-9, the Fas-receptor-containing death-inducing signaling complex for caspase-8 and the NLRP3-containing inflammasome for caspase-1. Caspase-2 has been one of the last caspase activation and recruitment domain (CARD)containing caspases for which no such activating platform was identified. Read et al. ${ }^{7}$ showed that caspase-2 is activated in a high-molecular-weight platform, the composition of which remained unknown. In an interaction screen exclusively containing various death domain-containing proteins performed in Jürg Tschopp's laboratory, PIDD was found to be a binding partner of receptor-interacting protein (RIP)-associated ICH-1/CED-3 homologous protein with a death domain (RAIDD), ${ }^{4}$ a known caspase-2 CARD interactor. Upon activation, PIDD recruited both RAIDD and caspase-2 forming a complex of about $700 \mathrm{kDa}$, which we called the PIDDosome (the name changed later to caspase-2 PIDDosome). ${ }^{8,9}$ Caspase-2 recruitment to the PIDDosome resulted in its processing in the p31, p19 and p12 subunits and in its activation, as monitored by pull-down experiments with biotin-VAD and by bimolecular fluorescence complementation., ${ }^{4,9}$
This suggested that the PIDD-RAIDD-caspase-2 complex indeed offered a platform for caspase-2 activation. Although both PIDD-C (residues 446-910) and PIDD-CC (residues 588-910) (see Figure 1) contain the DD and the putative oligomerization domain UPA and as such would be able to interact with the DD of RAIDD, ${ }^{6}$ PIDD-CC is the main mediator of caspase-2 activation. Indeed, immunoprecipitation of RAIDD specifically pulls down PIDD-CC and not PIDD-C in cells stably overexpressing full-length PIDD, showing that only PIDD-CC is part of the caspase-2 PIDDosome and is sufficient to drive caspase- 2 activation. ${ }^{6}$

The elucidation of the crystal structure of the complex formed by PIDD-DD and RAIDD-DD revealed a surprising core composed of a ring of five PIDD-DD on top of a ring of seven RAIDD-DD. ${ }^{10}$ Despite the asymmetry of the complex, all DDs are located in an equivalent environment. There are three types of interactions between the different DD, totalling eight different kinds of interface interactions. Mutating a residue that disrupts one of these interfaces results in the disruption of the complex assembly and in defective caspase2 processing. ${ }^{10}$ These data therefore support and confirm the hypothesis that the DD-containing protein PIDD, just like Apaf-1, forms the core moiety of a large oligomeric complex, which through the recruitment of an adaptor protein RAIDD allows proximity-induced activation of an initiator caspase.

Ligands or stimuli-activating PIDD remain as elusive as the ones activating caspase-2. Heat shock and DNA damage seem to be the best characterized PIDDosome stimuli, ${ }^{4,9,11-13}$ although there is still some debate as to whether heat shock really activates caspase-2. ${ }^{14,15}$ Recently, caspase-2 was shown to be activated by several new stimuli, suggesting its involvement in several additional pathways, such as apoptosis induced by endoplasmic reticulum stress or cytoskeletondisrupting agents, apoptosis induced by metabolic stress or pathogen-induced macrophage apoptosis. ${ }^{16-22}$ It is currently unknown whether these are true biological triggers for caspase-2 and/or whether PIDD and RAIDD are involved in these processes as well.

Even in response to DNA damage, the role of PIDD in apoptosis remains very controversial. PIDD-mediated activation of caspase-2 results only in particular cell types in cell death, while in others it leads to growth suppression coupled to sensitization to apoptosis. ${ }^{2,4,23,24}$ Cells stably expressing PIDD show a significant portion (50-75\%) of processed and activated endogenous caspase-2, but can survive despite showing a slowed division. Activated caspase-2, however, seems to potentiate the effects of pro-apoptotic agents, as PIDD-expressing HeLa cells appear to be much more prone to cell death (A Tinel, unpublished observations). Further characterization of PIDD-expressing HeLa cells revealed that they exhibited a constitutive partial mitochondrial outermembrane permeabilization (measured by 3,3'-dihexyloxacarbocyanine iodide (DiOC6) fluorescence), and a dramatic increase in cytochrome $c$ release upon treatment with apoptotic agents, leading to increased caspase- 9 and -3 activation. With the notable exception of the partial mitochondrial outer-membrane permeabilization, Bcl-2 expression inhibited caspase activation and cytochrome $c$ release. ${ }^{25}$ Caspase-9 deficiency however only mildly delayed PARP cleavage, but did not prevent any other of the above-cited 
observations. Interestingly, zVAD-fmk treatment for $24 \mathrm{~h}$ of PIDD-expressing HeLa cells did not prevent the partial mitochondrial outer-membrane potential loss, suggesting a non-enzymatic direct role of processed caspase-2 on the mitochondria as described previously (A Tinel, unpublished observations). ${ }^{26-28}$

Most importantly, mice deficient for PIDD or RAIDD could not confirm a role for PIDD in caspase-2-dependent apoptosis. ${ }^{23,29,30}$ The RAIDD-KO confirmed that PIDD-mediated apoptosis and growth suppression is RAIDD dependent, but only partially caspase-2 dependent, suggesting the existence of caspase-2-independent functions of PIDD. ${ }^{23}$ RAIDDdeficient mice undergo normal apoptosis and normal caspase-2 processing during cell death, questioning the role of RAIDD as a caspase-2 adaptor. Similar conclusions were drawn from the PIDD-deficient mice. No role for PIDD in p53- or cytotoxic agent-induced apoptosis could be demonstrated, and caspase-2 processing remained unaltered, questioning the role of PIDD in caspase-2 activation. ${ }^{29,30}$ The lack of any apoptotic phenotype in RAIDD- and PIDDdeficient mice is reminiscent of the absence of an apoptotic defect in caspase-2-deficient mice. ${ }^{25,30,31}$ This shows that just like for caspase-2, there is a discrepancy between observations carried out in cells with a transient deletion of PIDD or RAIDD-by small interfering RNA (siRNA) treatment-compared to cells/tissues with a more permanent deletion of PIDD or RAIDD-by shRNA or by generating the full knockout (KO) mouse. Several explanations may account for this. Although it is clear that there is a major difference between in vitro and in vivo obtained results, it seems likely that the PIDDRAIDD-caspase-2 pathway is prone to compensatory mechanisms and/or shows redundancy with other apoptotic pathways, as suggested previously for caspase- $2 / 9,{ }^{32}$ and as was clear in our in vitro studies as well (A Tinel, unpublished observations). We believe that the level of PIDD activation may also explain some discrepancies: while massive caspase-2 activation mediated by PIDD overexpression appears to be detrimental to the cell and to promote cell death, more modest activation of caspase-2 may lead to a different outcome, ${ }^{12,33}$ as will be described further. Finally, these contradictory results could also be explained by the existence of alternative molecular platform-activating caspase-2, independent of PIDD and RAIDD, with the PIDDosome being only implicated in caspase-2 activation under specific conditions.

The tools that have been used to monitor caspase-2 activity were for a very long time unsatisfactory and may also have led to some erroneous conclusions. ${ }^{9,34,35}$ PIDD-mediated caspase-2 activation was mainly examined by monitoring the processing of caspase-2 and the appearance of the p31 and p19 subunits. Considering that the processing is an insufficient marker of activation, ${ }^{9}$ as caspase- 3 was shown to cleave caspase-2 downstream of mitochondrial outer-membrane permeabilization ${ }^{36}$ without activating it, ${ }^{8}$ it would be interesting to monitor caspase-2 activation functionally or with biotinVAD pull-down in PIDD- and RAIDD-deficient cells to unequivocally address the involvement of PIDD in caspase2 activation. What is clear however is that most likely, in normal conditions, induction of apoptosis is not the main function of either PIDD or caspase-2. Indeed, further studies revealed that caspase-2-induced apoptosis was only detectable in cells in which specific mutations or deletions occurred (see further). Furthermore, the discovery of additional interaction partners of PIDD revealed the existence of additional PIDD complexes with novel functions.

\section{PIDD and NF- $\kappa$ B Activation: the NEMO PIDDosome}

While searching for new components of the PIDDosome, RIP1 and NEMO were found to interact with PIDD upon activation. ${ }^{37}$ Both proteins are major players in nuclear factor- $\kappa \mathrm{B}(\mathrm{NF}-\kappa \mathrm{B})$ signaling and had been implicated before in DNA damageinduced NF- $\kappa$ B activation. ${ }^{38-40}$ It was, however, unclear as to how they would link a nuclear signal (being DNA damage) with a cytosolic signaling complex (activation of the IKK complex). Janssens et al. ${ }^{37}$ revealed that upon genotoxic stress, PIDD translocates to the nucleus and forms a complex with RIP1 and NEMO, enabling NEMO sumoylation. This modification of NEMO had previously been shown to be essential for recognition of NEMO by the nuclear kinase ATM and for the induction of its subsequent phosphorylation and ubiquitination. ${ }^{40}$ This is sufficient to trigger NEMO export to the cytosol and to activate the IKK complex. ${ }^{40}$ While PIDD and RIP1 interact in a direct way via their DD, the interaction between NEMO and PIDD is mediated via RIP1, explaining why it is essential for genotoxic stress-induced $\mathrm{NF}-\kappa \mathrm{B}$ activation. $^{38}$ The role of PIDD in DNA damage-induced NF- $\kappa \mathrm{B}$ activation was confirmed by the analysis of the PIDD-KO animals (A Villunger, oral presentation, ECDO meeting, Ghent, Belgium, September 2010). Again, only a part of the PIDD protein could be found within the NEMO PIDDosome (Table 1). Despite the presence of a DD in both PIDD-C and PIDD-CC, co-immunoprecipitations with the DD of RIP1 revealed a specific interaction with PIDD-C only and not with PIDD-CC. ${ }^{37}$ Furthermore, the RIP1/NEMO and RAIDD/caspase-2 pathways induced by PIDD appeared to be mutually exclusive. This suggested that PIDD could act as a switchboard either activating a cell survival pathway in which PIDD-C, RIP1 and NEMO played an essential role or activating an apoptotic pathway in which PIDD-CC, RAIDD and caspase- 2 were the important factors. ${ }^{26}$ The sequential generation of two different PIDD fragments with two different functions appeared to be an essential regulatory step in PIDD functioning, as will be discussed further in the text.

\section{PIDD, Cell Cycle Arrest and DNA Repair: the PCNA and DNA-PKc PIDDosome}

Already from the first paper describing the PIDDosome, it seemed rather unlikely that its sole function would be induction of apoptosis alone. Despite constitutive PIDDosome formation and high levels of caspase-2 activation observed in cells overexpressing PIDD, those cells survived, and the existence of apoptosis-independent functions of PIDD such as DNA repair were already postulated in the first paper describing the PIDDosome. ${ }^{4}$ Large-scale immunoprecipitations with PIDD carried out in two different labs confirmed that most PIDD interactors appeared to be members from the DNA repair machinery and/or the cell cycle checkpoint machinery (C Du and A Villunger, personal communications). ${ }^{41}$ Consistent with this, it is known that both PIDD and caspase- 2 can 
Table 1 The PIDDosomes

\begin{tabular}{|c|c|c|c|c|}
\hline Platform & Stimulus & Components & Outcome & References \\
\hline $\begin{array}{l}\text { Caspase-2 } \\
\text { PIDDosome }\end{array}$ & $\begin{array}{l}\text { DNA damage } \\
\text { Heat Shock }\end{array}$ & $\begin{array}{l}\text { PIDD (PIDD-CC) } \\
\text { RAIDD } \\
\text { Caspase-2 }\end{array}$ & $\begin{array}{l}\text { Caspase-2 activation } \\
\text { Cell death } \\
\text { Drug resistance }\end{array}$ & $\begin{array}{l}\text { Tinel et al. }{ }^{4,6} \\
\text { Tu et al. } \\
\text { Oliver et al. }\end{array}$ \\
\hline $\begin{array}{l}\text { NEMO } \\
\text { PIDDosome }\end{array}$ & DNA damage & $\begin{array}{l}\text { PIDD (PIDD-C) } \\
\text { RIP1 } \\
\text { NEMO }\end{array}$ & $\begin{array}{l}\text { NEMO modifications } \\
\text { NF- } \kappa \mathrm{B} \text { activation }\end{array}$ & Janssens et al. ${ }^{37}$ \\
\hline $\begin{array}{l}\text { PCNA } \\
\text { PIDDosome }\end{array}$ & UV & $\begin{array}{l}\text { PIDD (PIDD-C) } \\
\text { PCNA } \\
\text { RFC5, RFC4 }\end{array}$ & $\begin{array}{l}\text { PCNA monoUb } \\
\text { DNA repair } \\
\text { Translesion DNA synthesis }\end{array}$ & Logette et al. ${ }^{41}$ \\
\hline $\begin{array}{l}\text { DNA-PKc } \\
\text { PIDDosome }\end{array}$ & DNA damage & $\begin{array}{l}\text { PIDD } \\
\text { RAIDD } \\
\text { DNA-PK }\end{array}$ & $\begin{array}{l}\text { Caspase- } 2 \text { phosphorylation } \\
\text { Cell cycle checkpoint } \\
\text { DNA repair }\end{array}$ & $\begin{array}{l}\text { C Du and A Villunger } \\
\text { (personal communications) }\end{array}$ \\
\hline
\end{tabular}

be found in the nucleus (constitutively in the case of caspase2 and upon DNA damage in the case of PIDD ${ }^{30,37}$ ) and that caspase-2 is the only caspase identified so far with a nuclear localization signal in its prodomain. ${ }^{42}$

Accumulating evidence from over the last few years point out that caspase-2 and PIDD might be implicated in pathways that act much earlier than DNA damage-induced apoptosis and are important for cell cycle control and DNA repair rather than cell death. In normal conditions, caspase-2-dependent apoptotic pathways upon DNA damage appear to be even suppressed by several independent mechanisms.

Consistent with a role for PIDD and caspase-2 in cell cycle control, both proteins have been linked to tumor suppression. Caspase-2 was found to be underexpressed in several cancers such as gastric carcinoma, ${ }^{43}$ metastatic brain tumors ${ }^{44}$ or acute childhood lymphoblastic leukemia T cells. ${ }^{45}$ Furthermore, low cellular levels of caspase-2 correlated with increased drug resistance in acute childhood lymphoblastic leukemia, indicative for a lost pro-apoptotic function of caspase-2. A very elegant study demonstrated that caspase-2 is a bona fide tumor suppression gene, with the loss of caspase-2 facilitating cellular transformation and increasing the tumorigenic potential of oncogene-transformed MEFs. ${ }^{46}$ This correlated with a reduced sensitivity to apoptosis induced by $\gamma$-irradiation and cytotoxic drugs. More caspase-2-deficient than control cells could be found in the cell cycle $24 \mathrm{~h}$ upon DNA damage, suggesting that an important cell cycle checkpoint might be lost. This was actually confirmed in a subsequent study (C Du and A Villunger, personal communications) where both PIDD and caspase-2 were shown to be involved in the maintenance of a DNA damage-induced cell cycle checkpoint, distinct from and operating in time later than the ATM checkpoint. Similarly, another study found a role for PIDD in growth arrest dependent on caspase-2 and p53. ${ }^{12}$ The role of PIDD in tumor suppression is less clear. Bradley et al. ${ }^{47}$ showed that PIDD levels varied highly in different oral squamous carcinoma cells (OSCC), but elevated levels positively correlated with a high apoptotic index in OSCC. This was consistent with data obtained in malignant melanoma where p53-dependent transcriptional gene products (such as PIDD and p21) were downregulated by $\mathrm{S} 100 \mathrm{~B}$, an inhibitor of $\mathrm{p} 53 .{ }^{48}$ siRNA transfections against S100B restored p53 and PIDD levels and increased p53-dependent apoptotic pathways, consistent with earlier papers on the role of PIDD in p53-dependent apoptosis. ${ }^{2}$ On the other hand, significantly increased expression of PIDD was found in renal cell carcinomas and in lung tumors. $^{33,49}$ In lung tumors, the increased PIDD levels correlated with the promotion of cell cycle arrest and the development of cisplatin-resistant tumors. ${ }^{33}$ The authors speculated that this could be related to the known prosurvival function of PIDD, ${ }^{37}$ but later found out that it was actually PIDD-CC, and not PIDD-C, which conferred drug resistance. In response to DNA damage, the caspase-2 PIDDosome facilitates caspase-2-mediated cleavage of Mdm2 and as such helps in the formation of an inhibitory p60 fragment of Mdm2 that blocks p53 ubiquitination. PIDD and caspase-2 expression leads to the stabilization of p53, which presumably in this case preferentially induces cell cycle arrest and DNA damage repair, rather than apoptosis, explaining the increased drug resistance. Finally, the authors suggested that this pathway is operational under reparable level of DNA damage only, as under higher levels of damage both fulllength and cleaved Mdm2 levels are reduced, again reflecting the fact that different doses of DNA damage might trigger opposing pathways in a cell.

PIDD and caspase-2 are not only linked to cell cycle control and tumor progression, but also to pathways of DNA repair. Several protein interactors of PIDD having a role in DNA replication and/or repair were identified (C Du and A Villunger, personal communications). ${ }^{41}$ The DNA-PKc PIDDosome, consisting of a complex of both PIDD and caspase-2 with DNA-PKc, revealed a role for PIDD and caspase-2 in nonhomologous end-joining repair mechanisms ( $\mathrm{C} \mathrm{Du}$ and $\mathrm{A}$ Villunger, personal communications) (Table 1). Of note, the relevance of some of these interactions remains to be formerly confirmed. The interaction between PIDD and DNA-PKc was confirmed by Logette et al. ${ }^{41}$ In addition, they detected almost all members of the proliferating cell nuclear antigen (PCNA)/ $\mathrm{RFC} /$ pol $\delta$ complex as PIDD-interacting partners, the PCNA PIDDosome (Table 1), and found a major role for PIDD in the monoubiquitination of PCNA upon DNA damage. PIDD overexpression or UV irradiation and concomitant PIDD induction triggers PCNA monoubiquitination upon release of p21. This allows the interaction of PCNA with low stringency DNA polymerases, such as pol $\eta$ initiating the so-called translesion DNA synthesis (TLS), a mechanism of DNA replication that escapes repair and allows cells to avoid replication arrest and subsequently apoptosis. Indeed, inhibition of PIDD was associated with increased apoptosis in response to UV-C, an observation that was very similar to the 
one made by Oliver et al., ${ }^{33}$ showing that PIDD deficiency led to enhanced apoptosis upon low doses of doxorubicin. This suggests again that under moderate levels of DNA damage, PIDD and caspase-2 are needed for DNA repair and replication, rather than apoptosis. Intriguingly, Sidi et al. ${ }^{50}$ described in both zebrafish and human tumor cells a mechanism that could account for this. In normal conditions, the checkpoint kinase 1 (Chk1) suppresses a caspase-2dependent apoptotic response that bypasses p53, Bcl-2 and caspase-3. Under conditions of Chk-1 inhibition, caspase-2dependent apoptotic programs are restored. This coincides well with the fact that Chk-1 inhibition sensitizes colon carcinoma HCT116 cells to lidamycin through abrogation of a G2/M checkpoint and induction of a caspase-2-dependent apoptotic pathway. In addition, a distinct mechanism of caspase-2 inhibition during mitosis has been described by Andersen et al. ${ }^{51}$ While caspase- 2 has been described as a major player in mitotic catastrophe, ${ }^{52}$ this is normally prevented by phosphorylation of caspase-2 on $\$ 340$ by Cdk1-cyclinB1. ${ }^{53}$ When endogenous caspase-2 was replaced with the S340A mutant, U2OS cells treated with nocodazole were found to undergo mitotic catastrophe much more readily. For PIDD, most likely, similar regulatory mechanisms are operational. We found that under low doses of $\gamma$-irradiation preferentially PIDD-C was induced, leading to activation of cell survival pathways, while under conditions of high doses of $\gamma$-irradiation or genotoxic drugs, PIDD-CC appeared to be the predominant fragment found and apoptotic pathways were initiated. ${ }^{6}$ Both pathways seemed to be mutually exclusive. ${ }^{37}$ In conclusion, these data show that under normal conditions and under reparable levels of DNA damage, PIDD and caspase-2 do not trigger cell death, but suppress apoptotic programs by various means. In these circumstances, their physiological function appears to be more related to mechanisms of DNA repair, DNA replication, cell cycle control and/or cell survival. Still, numerous papers show that PIDD and caspase-2 can initiate apoptotic pathways upon DNA damage, particularly in conditions of overexpression, or under high levels of DNA damage, most likely reflecting an extreme situation in the cell. As such, they might be involved in a mechanism ensuring the demise of cells in conditions where proper genome surveillance fails (e.g. in the case of Chk1 inhibition) or in cases of unreparable DNA damage. However, as the $\mathrm{KO}$ mice data do not support a role in apoptosis, the physiological relevance of this latter pathway is still under debate.

\section{Getting it All Together: fine-tuning PIDD Function}

The function of PIDD is controlled in a very tight manner by several different mechanisms, allowing a variety of different fragments with specific functions to be generated. PIDD expression levels are regulated at the transcriptional level and by alternative splicing, while its function is further regulated by autoproteolytic cleavage as well as through intra- and intermolecular protein interactions (Figure 2). PIDD stands for p53-induced protein with a $\mathrm{DD}^{2}$ and is strongly induced in a p53-dependent way or by inhibition of the inhibitor of apoptosis protein protein BRUCE. ${ }^{2,54,55}$ Still, at basal level, no correlation could be found between the p53 status of a cell and PIDD expression levels, showing that at least basal expression of PIDD is p53-independent. ${ }^{33}$ Besides, Cuenin et $a l .{ }^{56}$ showed that some DNA damage-inducing agents, like doxorubicin, do induce PIDD in a strict p53-dependent way, while others, like etoposide, appear to use alternative pathways for PIDD induction.

Four different isoforms of PIDD with slight sequence differences have been described so far. ${ }^{1,56,57}$ Isoform 1 is the full-length 910 amino acids form of PIDD, identified by Lin et $a l^{2}{ }^{2}$ Isoform 2, described as LRDD, ${ }^{1}$ lacks the 147 aminoterminal acids as well as 11 amino acids (580-590) compared with isoform 1. The deletion affects the second cleavage site of PIDD (S588), hampering formation of the PIDD-CC fragment. A third isoform has the same $\mathrm{N}$-terminal extension as isoform 1 , but contains a small deletion of 17 residues (705-721) compared with isoform 1. The three isoforms can

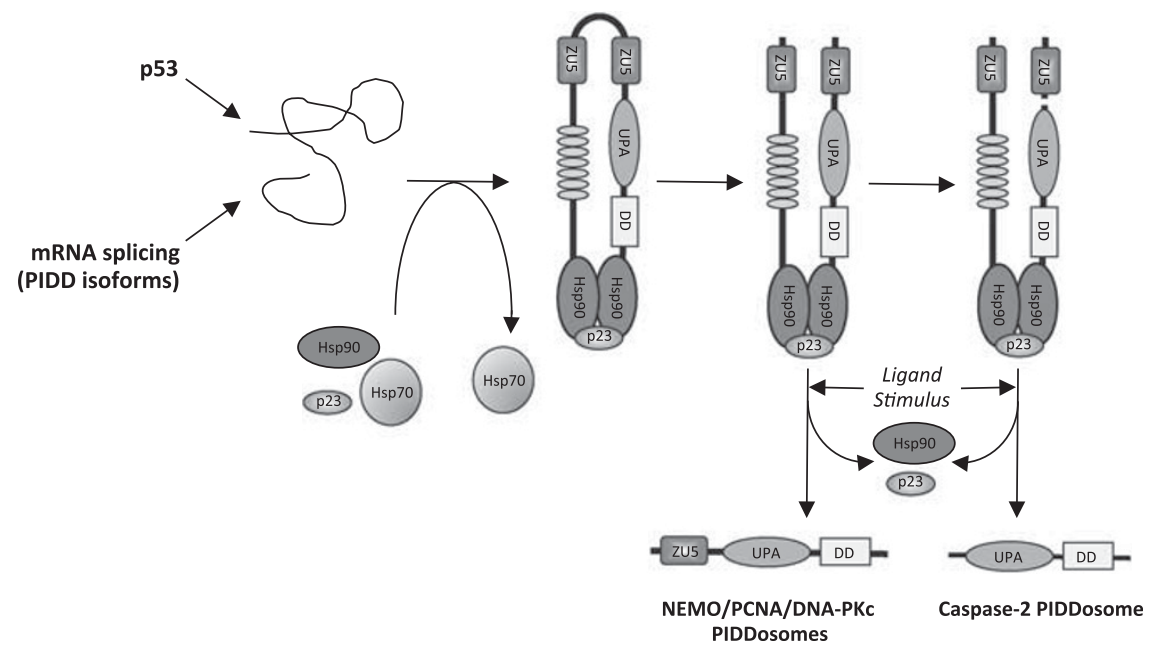

Figure 2 A schematic model of PIDD maturation and activation. PIDD isoforms are expressed constitutively or induced by p53. PIDD is synthesized as a $101 \mathrm{kDa}$ protein that requires $\mathrm{Hsp} 90$ binding for its conformation, maturation and stability. After its crucial involvement in PIDD processing, Hsp90 together with its co-chaperone p23 remains bound to PIDD and participates in PIDD activation, allowing the release of the active PIDD-C and PIDD-CC fragments. Isoform 2 does not generate PIDD-CC fragments owing to the absence of the S588 cleavage site 
activate $\mathrm{NF}-\kappa \mathrm{B}$ in response to genotoxic stress, but only isoform 1 has the capacity to activate caspase- 2 in an RAIDDdependent manner. Other small deletions in the same region confirmed the essential role of the UPA domain in caspase-2 activation (A Tinel, unpublished observations). Isoforms 1 and 3 are the most ubiquitously expressed isoforms, while isoform 2 shows - at least at tissue level-a highly restricted expression. At protein level, western blot analysis of endogenous PIDD suggests that the different PIDD isoforms are expressed at quite equivalent levels, especially when one considers the PIDD-C fragment. ${ }^{6,13,56}$ Upon DNA damage, only mRNA for isoform 3 is induced, while the two other isoforms are unaffected, as this isoform is unable to trigger apoptosis, this points again towards an important role for alternative PIDD functions upon DNA damage. ${ }^{56}$ Recently, a fourth isoform (PIDD4) has been described that lacks the LRR domain. ${ }^{57}$ Interestingly, this isoform is constitutively active and strongly induces apoptosis. This result is in agreement with previous observations that PIDD-LRR is more active (A Tinel, unpublished observations) ${ }^{6}$ and suggests that the LRR region may fulfill a regulatory role (see also further).

At the post-translational level, PIDD has also elaborated elegant ways to generate different active fragments. To accommodate with both the caspase-2- and NF- $\kappa$ B-activating function, PIDD can generate two docking platforms from a single protein by a cleavage at two different $\operatorname{sites}^{6}$ (Figure 2). Indeed, PIDD-C is the fragment that is involved in pro-survival and repair functions (TLS and NF- $\kappa$ B activation), while PIDDCC binds RAIDD and initiates caspase-2 activation. ${ }^{6,12,41}$ As suggested by the high homology between their cleavages sites, PIDD is cleaved in a manner similar to the nuclear pore proteins Nup98/96, an autoprocessing mechanism also shared by inteins. ${ }^{6,58-61}$ Characterization of PIDD processing showed that it is sequentially cleaved: in a first cleavage step, PIDD undergoes a cleavage at position S445, generating PIDD-N and PIDD-C, and in a second equivalent cleavage event, PIDD-C is further cleaved at S588 yielding the PIDD$\mathrm{CC}$ fragment ${ }^{6}$ (Figure 2). It remains unclear as to why the two cleavages do not occur simultaneously, and whether the timing of the autoprocessing steps can be regulated by exterior factors. However, nearly concomitant processing could be generated upon intense DNA damage leading to de novo PIDD synthesis. ${ }^{6}$ A limited amount of DNA breaks may initially only lead to the induction of the NF- $\kappa$ B-activating fragment PIDD-C, while more severe damage might simultaneously induce PIDD-C and PIDD-CC. This temporarily distinct generation of the prosurvival PIDD-C versus proapoptotic PIDD-CC fragment may govern the fate of the cell allowing time for cell survival, DNA repair and/or cell cycle control before cellular demise in case of exuberant DNA damage. The exact role of PIDD-N remains unclear, although it is thought to be a regulatory fragment. While PIDD-N can interact with both PIDD-C and PIDD-CC fragments, keeping all the fragments together even after the cleavage events, deletion or absence of the LRR leads to an increased activity (A Tinel, unpublished observations). ${ }^{6,57}$ LRRs are frequently found in pathogen recognition molecules, where they are supposed to act as the ligand-sensing part of the protein, their repetitive structure being ideal to allow rapid evolution of novel variants. In the unbound state, LRRs may be implicated in negative regulation. Thus, in Nod-like receptors (NLRs) LRRs are thought to fold back intramolecularly onto the NACHT (domain present in neuronal apoptosis inhibitory protein (NAIP), the major histocompatibility complex (MHC) class II transactivator (CIITA), HET-E and TP1) domain, preventing it from oligomerization and therefore keeping the molecule in an inactive, autorepressed state. ${ }^{62}$ Once the signal is recognized, the molecule undergoes a conformational change, allowing oligomerization and activation of the protein. It is possible that LRR in PIDD fulfill a similar function.

Another regulatory level is the proposed autoinhibitory role of the ZU5-UPA-DD domain. Wang et al. $^{3}$ demonstrated that in Unc5B, the ZU5 domain binds the DD adopting a closed conformation. Opening of this closed conformation could be achieved by binding of regulatory proteins to the ZU5 or DD. Alternatively, one could postulate that the autoproteolytic cleavage of PIDD between the ZU5 and the DD may release the autoinhibition of the DD and could allow for a fully active PIDD-CC fragment. This mechanism remains to be firmly established in PIDD as no interaction between PIDD ZU5 and its DD could be detected (A Tinel, unpublished observations).

Finally, PIDD is regulated by a number of proteins that controls its stability and conformation, as well as its activation. Indeed, in resting cells, PIDD can be readily found in a constitutively autoprocessed form. This suggests the requirement for an additional stimulus or the removal of a regulatory protein for the full activation and formation of the different complexes. A search for such regulatory proteins led to the identification of heat-shock protein 90 (Hsp90) as a binding partner for PIDD. ${ }^{13}$ In unstimulated cells, the Hsp90, as well as its co-chaperone p23, is bound to PIDD, and is essential for its stability, and optimal autoprocessing capacity by supporting its conformation (Figure 2). In addition to its strictly chaperone function, Hsp90 plays a crucial role in PIDD activation and function. 9,13 Activation of PIDD is associated with Hsp90 release, resulting in signaling-competent PIDDosomes (such as nuclear PIDD) that no longer interact with Hsp90. Interestingly, Hsp90 interaction with PIDD is required for PIDD activation as measured by caspase-2 and NF- $\kappa \mathrm{B}$ activation. However, although Hsp90 is required for PIDD autocleavage, stability and function, it is not a part of the final signaling complex. ${ }^{13}$ PIDD and caspase- 2 have been shown to be activated by heat shock, ${ }^{9,11}$ and therefore it is possible that Hsp90 could actually function as a heat sensor. Many LRR-containing proteins, such as NLRs, require Hsp90 for their function and it has been suggested that chaperones support the less stable, but functionally advantageous LRRs in their client proteins. ${ }^{63,64}$ In addition, $\mathrm{Hsp} 70$ and CHIP were found to interact with PIDD. This was found to be independent of PIDD activation status and we proposed that Hsp70 may be needed to tag PIDD for degradation as part of a negative feedback loop on PIDD activity.

\section{Concluding Remarks}

As might be clear from the previous parts, there are still many open questions in the PIDD field. Although initial studies suggested a role for PIDD in the activation of caspase-2 and the induction of DNA damage-induced apoptosis, this has been challenged by the phenotyping of PIDD- and 
RAIDD-deficient mice. A picture is emerging in which PIDD and/or caspase-2 seem to be involved in pathways acting much earlier than apoptosis, at the level of cell cycle control, DNA repair and/or NF- $\kappa$ B activation, while PIDD-dependent apoptosis seems only induced in extreme circumstances (such as PIDD or caspase-2 overexpression). More research is clearly needed to address whether PIDD and caspase-2 are operating in the same pathway in these novel functions, or whether they can be disconnected, just like in the NF- $\kappa \mathrm{B}-$ activating PIDD-C/RIP1/NEMO complex. In addition, it remains to be demonstrated if and how PIDD is triggered by DNA damage. These experiments will be essential for our further understanding of this fascinating molecule.

\section{Finally ...}

This work was all carried out in Jürg Tschopp lab and to both of us it felt odd not to be able anymore to hand the paper over to him when we came to the concluding remarks section. Because this is what he loved to write and this is where he was particularly good in. He loved to speculate about the bigger picture and had an amazingly broad view of how protein functions would be linked to other protein functions, to different pathways in a cell and to the biology of an organism in general. This is also why it was so fascinating to work with him. $A$ result obtained in the evening, and the next morning Jürg would immediately start drawing the most remarkable schemes and models of what it could mean in terms of cancer, inflammation, apoptosis or whatever process inside the cell.

We would like to take this occasion to thank him enormously for everything he taught us. He was a fantastic mentor and the scientific community has lost a great scientist. We hope investigation in this field will continue in his memory.

\section{Conflict of Interest}

The authors declare no conflict of interest.

Acknowledgements. We thank all members of the group Tschopp who over the years helped in unraveling the functions of this fascinating protein PIDD: S Lippens, S Cuenin, E Logette, M Eckert, and K Ludigs. We would further like to thank N Aebi, S Hertig, and C Mattmann for their excellent technical support and all group Tschopp members for the fantastic discussions over the years.

1. Telliez JB, Bean KM, Lin LL. LRDD, a novel leucine rich repeat and death domain containing protein. Biochim Biophys Acta 2000; 1478: 280-288.

2. Lin Y, Ma W, Benchimol S. Pidd, a new death-domain-containing protein, is induced by p53 and promotes apoptosis. Nat Genet 2000; 26: 122-127.

3. Wang R, Wei Z, Jin H, Wu H, Yu C, Wen W et al. Autoinhibition of UNC5b revealed by the cytoplasmic domain structure of the receptor. Mol Cell 2009; 33: 692-703.

4. Tinel A, Tschopp J. The PIDDosome, a protein complex implicated in activation of caspase-2 in response to genotoxic stress. Science 2004; 304: 843-846.

5. Pick R, Badura S, Bosser S, Zornig M. Upon intracellular processing, the C-terminal death domain-containing fragment of the $\mathrm{p53}$-inducible PIDD/LRDD protein translocates to the nucleoli and interacts with nucleolin. Biochem Biophys Res Commun 2006; 349: 1329-1338.

6. Tinel A, Janssens S, Lippens S, Cuenin S, Logette E, Jaccard B et al. Autoproteolysis of PIDD marks the bifurcation between pro-death caspase-2 and pro-survival NF-kappaB pathway. EMBO J 2007; 26: 197-208.

7. Read SH, Baliga BC, Ekert PG, Vaux DL, Kumar S. A novel Apaf-1-independent putative caspase-2 activation complex. J Cell Biol 2002; 159: 739-745.

8. Baliga BC, Read SH, Kumar S. The biochemical mechanism of caspase-2 activation. Cell Death Differ 2004; 11: 1234-1241.
9. Bouchier-Hayes L, Oberst A, McStay GP, Connell S, Tait SW, Dillon CP et al. Characterization of cytoplasmic caspase-2 activation by induced proximity. Mol Cell 2009; 35: $830-840$.

10. Park HH, Logette E, Raunser S, Cuenin S, Walz T, Tschopp J et al. Death domain assembly mechanism revealed by crystal structure of the oligomeric PIDDosome core complex. Cell 2007; 128: 533-546.

11. Tu S, McStay GP, Boucher LM, Mak T, Beere HM, Green DR. In situ trapping of activated initiator caspases reveals a role for caspase-2 in heat shock-induced apoptosis. Nat Cell Biol 2006; 8: 72-77.

12. Oliver TG, Meylan E, Chang GP, Xue W, Burke JR, Humpton TJ et al. Caspase-2-mediated cleavage of Mdm2 creates a p53-induced positive feedback loop. Mol Cell 2011; 43: 57-71.

13. Tinel A, Eckert MJ, Logette E, Lippens S, Janssens S, Jaccard B et al. Regulation of PIDD auto-proteolysis and activity by the molecular chaperone Hsp90. Cell Death Differ 2011; 18: $506-515$.

14. Milleron RS, Bratton SB. Heat shock induces apoptosis independently of any known initiator caspase-activating complex. J Biol Chem 2006; 281: 16991-17000.

15. Shelton SN, Dillard CD, Robertson JD. Activation of caspase-9, but not caspase-2 or caspase-8, is essential for heat-induced apoptosis in Jurkat cells. J Biol Chem 2010; 285 : 40525-40533

16. Krumschnabel G, Sohm B, Bock F, Manzl C, Villunger A. The enigma of caspase-2: the laymen's view. Cell Death Differ 2009; 16: 195-207.

17. Kumar S. Caspase 2 in apoptosis, the DNA damage response and tumour suppression: enigma no more? Nat Rev Cancer 2009; 9: 897-903.

18. Chen F, Ding X, Ding Y, Xiang Z, Li X, Ghosh D et al. Proinflammatory caspase-2-mediated macrophage cell death induced by a rough attenuated Brucella suis strain. Infect Immun 2011; 79: 2460-2469.

19. Chen $F$, He Y. Caspase-2 mediated apoptotic and necrotic murine macrophage cell death induced by rough Brucella abortus. PLoS One 2009; 4: e6830.

20. Kitevska T, Spencer DM, Hawkins CJ. Caspase-2: controversial killer or checkpoint controller? Apoptosis: An International Journal on Programmed Cell Death 2009; 14 : 829-848

21. Jesenberger V, Procyk KJ, Yuan J, Reipert S, Baccarini M. Salmonella-induced caspase-2 activation in macrophages: a novel mechanism in pathogen-mediated apoptosis. $J$ Exp Med 2000; 192: 1035-1046.

22. Vakifahmetoglu-Norberg H, Zhivotovsky B. The unpredictable caspase-2: what can it do? Trends Cell Biol 2010; 20: 150-159.

23. Berube C, Boucher LM, Ma W, Wakeham A, Salmena L, Hakem R et al. Apoptosis caused by p53-induced protein with death domain (PIDD) depends on the death adapter protein RAIDD. Proc Natl Acad Sci USA 2005; 102: 14314-14320.

24. Baptiste-Okoh N, Barsotti AM, Prives C. A role for caspase 2 and PIDD in the process of p53-mediated apoptosis. Proc Natl Acad Sci USA 2008; 105: 1937-1942.

25. O'Reilly LA, Ekert $P$, Harvey N, Marsden V, Cullen L, Vaux DL et al. Caspase-2 is not required for thymocyte or neuronal apoptosis even though cleavage of caspase-2 is dependent on both Apaf-1 and caspase-9. Cell Death Differ 2002; 9: 832-841.

26. Enoksson M, Robertson JD, Gogvadze V, Bu P, Kropotov A, Zhivotovsky B et al. Caspase2 permeabilizes the outer mitochondrial membrane and disrupts the binding of cytochrome c to anionic phospholipids. J Biol Chem 2004; 279: 49575-49578.

27. Robertson JD, Enoksson M, Suomela M, Zhivotovsky B, Orrenius S. Caspase-2 acts upstream of mitochondria to promote cytochrome $c$ release during etoposide-induced apoptosis. J Biol Chem 2002; 277: 29803-29809.

28. Robertson JD, Gogvadze V, Kropotov A, Vakifahmetoglu H, Zhivotovsky B, Orrenius S. Processed caspase-2 can induce mitochondria-mediated apoptosis independently of its enzymatic activity. EMBO Rep 2004; 5: 643-648.

29. Kim IR, Murakami K, Chen NJ, Saibil SD, Matysiak-Zablocki E, Elford AR et al. DNA damage- and stress-induced apoptosis occurs independently of PIDD. Apoptosis: An International Journal on Programmed Cell Death 2009; 14: 1039-1049.

30. Manzl C, Krumschnabel G, Bock F, Sohm B, Labi V, Baumgartner F et al. Caspase-2 activation in the absence of PIDDosome formation. J Cell Biol 2009; 185: 291-303.

31. Bergeron L, Perez Gl, Macdonald G, Shi L, Sun Y, Jurisicova A et al. Defects in regulation of apoptosis in caspase-2-deficient mice. Genes Dev 1998; 12: 1304-1314.

32. Troy CM, Rabacchi SA, Hohl JB, Angelastro JM, Greene LA, Shelanski ML. Death in the balance: alternative participation of the caspase-2 and -9 pathways in neuronal death induced by nerve growth factor deprivation. J Neurosci 2001; 21: 5007-5016.

33. Oliver TG, Mercer KL, Sayles LC, Burke JR, Mendus D, Lovejoy KS et al. Chronic cisplatin treatment promotes enhanced damage repair and tumor progression in a mouse model of lung cancer. Genes Dev 2010; 24: 837-852.

34. Bouchier-Hayes L, Green DR. Real time with caspase-2. Cell Cycle 2010; 9: 12-13.

35. McStay GP, Salvesen GS, Green DR. Overlapping cleavage motif selectivity of caspases: implications for analysis of apoptotic pathways. Cell Death Differ 2008; 15: 322-331.

36. Slee EA, Harte MT, Kluck RM, Wolf BB, Casiano CA, Newmeyer DD et al. Ordering the cytochrome c-initiated caspase cascade: hierarchical activation of caspases-2, -3, -6, -7, -8 , and -10 in a caspase-9-dependent manner. J Cell Biol 1999; 144: 281-292.

37. Janssens S, Tinel A, Lippens S, Tschopp J. PIDD mediates NF-kappaB activation in response to DNA damage. Cell 2005; 123: 1079-1092.

38. Hur GM, Lewis J, Yang Q, Lin Y, Nakano H, Nedospasov S et al. The death domain kinase RIP has an essential role in DNA damage-induced NF-kappa B activation. Genes Dev 2003; 17: 873-882 
39. Huang TT, Wuerzberger-Davis SM, Seufzer BJ, Shumway SD, Kurama T, Boothman DA et al. NF-kappaB activation by camptothecin. A linkage between nuclear DNA damage and cytoplasmic signaling events. J Biol Chem 2000; 275: 9501-9509.

40. Huang TT, Wuerzberger-Davis SM, Wu ZH, Miyamoto S. Sequential modification of NEMO/IKKgamma by SUMO-1 and ubiquitin mediates NF-kappaB activation by genotoxic stress. Cell 2003; 115: 565-576.

41. Logette E, Schuepbach-Mallepell S, Eckert MJ, Leo XH, Jaccard B, Manzl C et al. PIDD orchestrates translesion DNA synthesis in response to UV irradiation. Cell Death Differ 2011; 18: 1036-1045.

42. Baliga BC, Colussi PA, Read SH, Dias MM, Jans DA, Kumar S. Role of prodomain in importin-mediated nuclear localization and activation of caspase-2. J Biol Chem 2003; 278: 4899-4905.

43. Yoo NJ, Lee JW, Kim YJ, Soung YH, Kim SY, Nam SW et al. Loss of caspase-2, -6 and -7 expression in gastric cancers. Apmis 2004; 112: 330-335.

44. Zohrabian VM, Nandu H, Gulati N, Khitrov G, Zhao C, Mohan A et al. Gene expression profiling of metastatic brain cancer. Oncol Rep 2007; 18: 321-328.

45. Holleman A, den Boer ML, Kazemier KM, Beverloo HB, von Bergh AR, Janka-Schaub GE et al. Decreased PARP and procaspase-2 protein levels are associated with cellular drug resistance in childhood acute lymphoblastic leukemia. Blood 2005; 106: 1817-1823.

46. Ho LH, Taylor R, Dorstyn L, Cakouros D, Bouillet $\mathrm{P}$, Kumar $\mathrm{S}$. A tumor suppressor function for caspase-2. Proc Natl Acad Sci USA 2009; 106: 5336-5341.

47. Bradley G, Tremblay S, Irish J, MacMillan C, Baker G, Gullane P et al. The expression of p53-induced protein with death domain (Pidd) and apoptosis in oral squamous cell carcinoma. Br J Cancer 2007; 96: 1425-1432.

48. Lin J, Yang Q, Wilder PT, Carrier F, Weber DJ. The calcium-binding protein S100B downregulates p53 and apoptosis in malignant melanoma. J Biol Chem 2010; 285: 27487-27498.

49. Heikaus S, Pejin I, Gabbert HE, Ramp U, Mahotka C. PIDDosome expression and the role of caspase-2 activation for chemotherapy-induced apoptosis in RCCs. Cell Oncol 2010; 32: 29-42.

50. Sidi S, Sanda T, Kennedy RD, Hagen AT, Jette CA, Hoffmans R et al. Chk1 suppresses a caspase-2 apoptotic response to DNA damage that bypasses p53, Bcl-2, and caspase-3. Cell 2008; 133: 864-877.

51. Andersen JL, Johnson CE, Freel CD, Parrish AB, Day JL, Buchakjian MR et al. Restraint of apoptosis during mitosis through interdomain phosphorylation of caspase-2. EMBO 2009; 28: 3216-3227.
52. Castedo M, Perfettini JL, Roumier T, Valent A, Raslova $\mathrm{H}$, Yakushijin $\mathrm{K}$ et al. Mitotic catastrophe constitutes a special case of apoptosis whose suppression entails aneuploidy. Oncogene 2004; 23: 4362-4370.

53. Andersen JL, Kornbluth S. A cut above the other caspases. Mol Cell 2009; 35 733-734.

54. Seth R, Yang C, Kaushal V, Shah SV, Kaushal GP. P53-dependent caspase-2 activation in mitochondrial release of apoptosis-inducing factor and its role in renal tubular epithelial cell injury. J Biol Chem 2005; 280: 31230-31239.

55. Ren J, Shi M, Liu R, Yang QH, Johnson T, Skarnes WC et al. The Birc6 (Bruce) gene regulates p53 and the mitochondrial pathway of apoptosis and is essential for mouse embryonic development. Proc Natl Acad Sci USA 2005; 102: 565-570.

56. Cuenin S, Tinel A, Janssens S, Tschopp J. p53-induced protein with a death domain (PIDD) isoforms differentially activate nuclear factor-kappaB and caspase-2 in response to genotoxic stress. Oncogene 2008; 27: 387-396.

57. Huang L, Han D, Yang X, Qin B, Ji G, Yu L. PIDD4, a novel PIDD isoform without the LRR domain, can independently induce cell apoptosis in cytoplasm. Biochem Biophys Res Commun 2011; 407: 86-91.

58. Rosenblum JS, Blobel G. Autoproteolysis in nucleoporin biogenesis. Proc Natl Acad Sci USA 1999; 96: 11370-11375.

59. Hodel AE, Hodel MR, Griffis ER, Hennig KA, Ratner GA, Xu S et al. The three-dimensional structure of the autoproteolytic, nuclear pore-targeting domain of the human nucleoporin Nup98. Mol Cell 2002; 10: 347-358.

60. Paulus $\mathrm{H}$. Protein splicing and related forms of protein autoprocessing. Annu Rev Biochem 2000; 69: 447-496.

61. Perler FB, Xu MQ, Paulus H. Protein splicing and autoproteolysis mechanisms. Curr Opin Chem Biol 1997; 1: 292-299.

62. Martinon F, Tschopp J. NLRs join TLRs as innate sensors of pathogens. Trends Immunol 2005; 26: 447-454.

63. Mayor A, Martinon F, De Smedt T, Petrilli V, Tschopp J. A crucial function of SGT1 and HSP9O in inflammasome activity links mammalian and plant innate immune responses. Nat Immunol 2007; 8: 497-503.

64. Stuttmann J, Parker JE, Noel LD. Staying in the fold: the SGT1/chaperone machinery in maintenance and evolution of leucine-rich repeat proteins. Plant Signal Behav 2008; 3 283-285. 